\title{
Metabolomics study on model rats of chronic obstructive pulmonary disease treated with Bu-Fei Jian-Pi
}

\author{
JIANSHENG LI ${ }^{1 *}$, LIPING YANG $^{1 *}$, YA LI $^{2}$, YANGE TIAN ${ }^{2}$, SUYUN LI $^{2}$, \\ SULI JIANG $^{2}$, YING WANG ${ }^{2}$ and XINMIN LI ${ }^{2}$ \\ ${ }^{1}$ Basic Discipline of Integrated Chinese and Western Medicine, Henan University of Traditional Chinese Medicine, \\ Zhengzhou, Henan 450008; ${ }^{2}$ Respiratory Disease Institute, First Affiliated Hospital of \\ Henan University of Traditional Chinese Medicine, Zhengzhou, Henan 450000, P.R. China
}

Received February 4, 2014; Accepted September 12, 2014

DOI: $10.3892 / \mathrm{mmr} .2014 .2843$

\begin{abstract}
The therapeutic effect of Traditional Chinese Medicine (TCM) on chronic obstructive pulmonary disease (COPD) has been know for numerous years; however, the mechanism of action of the beneficial effects of TCM remains to be elucidated. The present study aimed to investigate the molecular mechanisms of COPD through metabolomic analysis as well as explore the targets and intervention mechanisms of TCM therapy using the common TCM granules Bu-Fei Jian-Pi. COPD rat models were established using smoke inhalations and recurrent bacterial infections. Rats were then divided into three groups as follows: A1, control healthy rats; B1, COPD model; and D1, Bu-Fei Jian-Pi-treated COPD rats. Following administration of the medicine, the metabolomic profile of the lung tissue of rats in each group was assessed using high-performance liquid chromatography/quadrupole-time-of-flight mass spectrometry. The results demonstrated that there was a significanlty different spectrum of metabolites in the lung tissue of the model group compared to that of the control group as well as the $\mathrm{Bu}-\mathrm{Fe}$ Jian-Pi-treated COPD group; in addition, following treatment with Bu-Fei Jian-Pi, the metabolites of COPD rats were comparable with those of the control. Notable changes were observed in 31 metabolites between the Bu-Fei Jian-Pi-treated group and the model group; however, there were 13 comparable metabolites between the Bu-Fei Jian-Pi and control groups as well as the model and control groups. Eleven metabolites showed a negative fold change in the Bu-Fei Jian-Pi-treated
\end{abstract}

Correspondence to: Professor Jiansheng Li, Basic Discipline of Integrated Chinese and Western Medicine, Henan University of Traditional Chinese Medicine, No. 1 Jinshui Road, Zhengzhou, Henan 450008, P.R. China

E-mail: bioylp@126.com

${ }^{*}$ Contributed equally

Key words: chronic obstructive pulmonary disease, Traditional Chinese Medicine pattern, Bu-Fei Jian-Pi granules, metabolites groups compared to concentrations in the model group; however, minimal changes were observed in phenylpyruvic acid and $\alpha$-D-fucose expression. In conclusion, the results of the present study demonstrated that Bu-Fei Jian-Pi granules had beneficial effects on measured outcomes in a rat model of stable COPD, indicated by a significantly different spectrum of metabolites. This therefore indicated that the metabolites which had significantly altered expression in the model group compared with that of the control and Bu-Fei Jian-Pi-treated groups may be potential biomarkers of COPD.

\section{Introduction}

Chronic obstructive pulmonary disease (COPD) is an increasingly prevalent disorder worldwide, which has high mortality rates and produces a severe economic burden (1). It has been predicted that by 2020 , COPD may become the third major cause of mortality in the world (1); in addition, in China alone, $\sim 65$ million mortalities due to COPD were estimated between 2003 and 2033 (2). It is therefore essential that novel mechanisms for the treatment and control of COPD are established. The risk factors of COPD include smoking, air pollution and solid-fuel use (2). Previous studies have provided evidence for the use of inhaled glucocorticosteroids, short- and long-acting bronchodilators as well as low-dose, slow-release theophylline being effective for the treatment of COPD (3); however, the adverse effects of therapeutic agents are difficult to avoid (4).

Traditional Chinese Medicine (TCM) has been used for numerous years for the treatment of COPD and is reported to have markedly advantageous effects on reducing the frequency of acute exacerbations and improving the quality of life of patients $(5,6)$. However, the molecular mechanisms of COPD and the beneficial effects of TCM remain to be elucidated; therefore, it is difficult to define the efficacy and advantages of TCM in the treatment of COPD (7). The TCM pattern is a set of priciples used to classify a disease according to a group of symptoms and summarize the patients' condition at various stages of the disease process (8). It was suggested that TCM patterns may be used to study COPD in the clinic. A previous study indicated that COPD exhibited three common TCM patterns, each of which responded to one specific herbal therapy (9). The aim of the present study was to investigate the molecular mechanisms 
of COPD through metabolomic analysis as well as explore the targets and intervention mechanisms of the common TCM granules Bu-Fei Jian-Pi in a rat model of stable COPD.

\section{Materials and methods}

Animal models. All animal handling and procedures were performed in accordance with the Guide for the Care and Use of Laboratory Animals (National Institutes of Health, Bethesda, MD, USA), following approval from the Institutional Animal Care and Use Committee at Henan University of Traditional Chinese Medicine (Henan, China). All surgeries were performed aseptically, with the minimal amount of pain and discomfort possible.

A total of 60 male and 60 female Sprague-Dawley rats (200-240 g; Beijing Laboratory Animal Research Center, Beijing, China) were each divided at random into three groups. Rats in the control group $(n=40)$ and model group $(n=40)$ were injected subcutaneously with $3.5 \mathrm{ml} / \mathrm{kg}$ physiological saline $(0.9 \% \mathrm{wt} / \mathrm{vol} \mathrm{NaCl})$. The treatment group $(\mathrm{n}=40)$ was injected subcutaneously with a solution of Bu-Fei Jian-Pi granules twice a day for 12 weeks. The volumes of TCM or saline were adjusted according to the body weight of each individual animal. The rats were kept in a 12-h light/dark cycle with food and water available ad libitum. At week 32, $\sim 24 \mathrm{~h}$ following the final injection, rats were administered anesthetic intraperitoneally with $50 \mathrm{mg} / \mathrm{kg}$ ketamine (Fujian Gutian Pharmaceutical Co., Ltd., Fujian, China) and sacrificed by cervical dislocation, the brains were then removed immediately. A thoracotomy was then performed in order to excise the superior, midle and inferior lobes of the right lung, the specimens were then snap-frozen in liquid nitrogen and stored at $-80^{\circ} \mathrm{C}$ until further use. This process was typically performed within 5-10 min following sacrification to limit post-mortem changes in the metabolite content of the samples.

Bacteria.Klebsiella pneumoniae (strain no. 46116) was provided by the National Center for Medical Culture Collection attached to the National Institutes for Food and Drug Control (Bejing, China). The concentration was adjusted to $6 \times 10^{8}$ colony forming units $(\mathrm{CFU}) / \mathrm{ml}$ prior to administration.

TCM and medicine. The Bu-fei Jian-Pi TCM therapy consisted of: $15 \mathrm{~g}$ radix Codonopsis dangshen; $15 \mathrm{~g}$ radix Astragali seu Hedysari huangqi; 12 g Poria fuling; 9 g bulbus Fritillariae Cirrhosae Chuanbeimu; 12 g Lumbricus dilong; and 9 g Pericarpium Citri Reticulatae chenpi. All herbs were prepared and provided by the Clinical Pharmacology Research Base of the First Affiliated Hospital of Henan University of TCM (Henan, China), a state TCM clinical research base. Prior to use, compounds were extracted and stored at $4^{\circ} \mathrm{C}$.

Constructing a stable rat model of COPD. Rat models of COPD were induced using smoke inhalations and recurrent bacterial infections. Rats were administered $0.1 \mathrm{ml}$ Klebsiella pneumoniae solution $\left(6 \times 10^{8} \mathrm{CFU} / \mathrm{ml}\right)$ through dripping into the nasal cavity every five days for eight weeks. Hongqiqu filter cigarettes consisting of: Flue-cured tobacco; $14 \mathrm{mg}$ tar; $1.2 \mathrm{mg}$ nicotine and $15 \mathrm{mg}$ carbon monoxide, were provided by the Henan Anyang Cigarette Factory (Henan, China) and were used for inducing stable COPD in rat models. The inhalations lasted for 30 minutes each time, three times a day with three-hour intervals between each inhalation. The inhalation lasted for 12 weeks in total. For each inhalation in weeks one and two, eight cigarettes were used and in weeks 3-12, 15 cigarettes were used. The COPD rat models generated were stable and suitable for the evaluation of the effects of the drug.

Grouping and medication. Rats were divided into groups as follows: A1, control group; B1, model group; and D1, Bu-Fei Jian-Pi-treated group D1. On the first day of the 9th week, groups A1 and B1 were orally administered with distilled water twice a day. The D1 group was administered $5 \mathrm{mg} / \mathrm{kg} /$ day under identical conditions. Treatment continued daily until the twentieth week, half of the rats were sacrificed at 20 weeks in order to study the short-term effects of the TCM and the other half of the rats were then kept until week 32 and sacrificed in order to determine the long-term effects of the TCM.

Content measurement and data collection. Lung tissue was collected in order to perform liquid chromatography-mass spectrometry (LC-MS) metabolic analysis.

Lung tissue was removed from the liquid nitrogen $\left(-80^{\circ} \mathrm{C}\right)$ and immediately cut into samples of $60 \pm 5 \mathrm{mg}$. Chromatographic methanol (Fujian Gutian Pharmaceutical Company) was pre-cooled to $-20^{\circ} \mathrm{C}$ and added to each sample $(200 \mu 1)$. A TissueLyzer II was used to homogenize the tissue $(30 \mathrm{~Hz}$; three minutes). The homogenized tissue solution was injected with $200 \mu \mathrm{l}$ chloroform (Fujian Gutian Pharmaceutical Company) and $300 \mu \mathrm{l}$ methanol (Fujian Gutian Pharmaceutical Company). Following centrifugation at $4^{\circ} \mathrm{C}$ for $15 \mathrm{~min}(8,000 \mathrm{x} \mathrm{g})$, Liquid supernatant $(800 \mu \mathrm{l})$ was then transferred into an Eppendorf tube. Methanol $(500 \mu \mathrm{l})$ was then added to the residue and centrifuged under identical conditions, the resultant supernatant $(500 \mu 1)$ was then transferred into the Eppendorf tube containing the former $800-\mu 1$ supernatant. Subsequently, $500 \mu 1$ mixed supernatant was removed and the solvent was removed under streaming nitrogen. The mixed supernantant was then reconstituted by adding $100 \mu \mathrm{l}$ aqueous methanol solution (1:1) and oscillated.

Following centrifugation under identical conditions, the supernatant was subjected to high-performance liquid chromatography/quadrupole-time-of-flight mass spectrometry (HPLC/QToF-MS), set up as follows: Agilent 1200 series HPLC system with Agilent Poroshell SB-C18 Reversed-Phase Column $(2.1 \times 50 \mathrm{~mm} ; 2.7 \mu \mathrm{l})$ and the Agilent 6520 series QtoF-MS mass spectrometer (Agilent Technologies, Inc., Santa Clara, CA, USA). Mobile phase (flow rate, $0.3 \mathrm{ml} / \mathrm{min}$; injection volume, $5 \mu \mathrm{l}$ ) for the ultrapure solution contained: A, $0.1 \%$ formic acid; and B, an acetonitrile solution of $0.1 \%$ formic acid. The chromatographic elution procedure was performed at $40^{\circ} \mathrm{C}$ as follows: $0 \mathrm{~min}, 1 \% \mathrm{~B} ; 1 \mathrm{~min}, 1 \% \mathrm{~B} ; 3 \mathrm{~min}, 45 \% \mathrm{~B}$; 9 min, $80 \%$ B; 11 min, $100 \%$ B; 18 min, $100 \%$ B; 19 min, $1 \%$ B; $25 \mathrm{~min}, 1 \% \mathrm{~B}$. MS settings were as follows: Ion source, Dual ESI; ionization mode, ESI+; desolvation and atomization gas, high-purity nitrogen $>99.999 \%$ ); gas flow rate, 10 1/min; $330^{\circ} \mathrm{C}$; spray fog pressure, $40 \mathrm{psi}$; capillary voltage, 4,000 V; capillary fragmentor voltage, $135 \mathrm{~V}$; skimmer voltage, $65 \mathrm{~V}$; MS acquisition range, 100-1,000 Da (with online calibration mode 


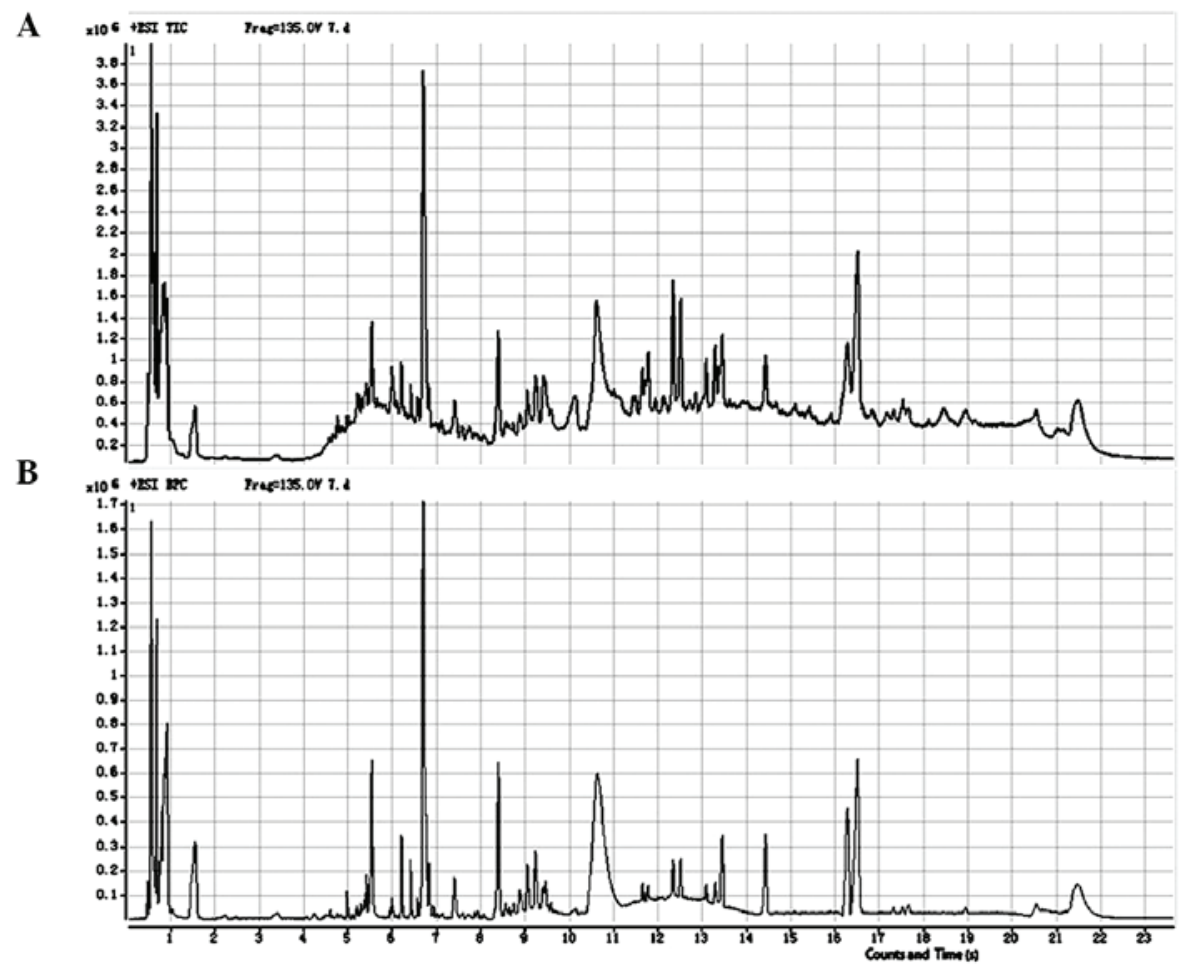

Figure 1. (A) Total ion chromatogram and (B) basepeak chromatogram of representative lung tissue samples of the control group. The R platform was then used to process the raw data with the XCMS package to obtain information about the baseline filter, peak identification, retention times and peak alignment. The three-dimensional structural data was then organized into a two-dimensional matrix of data, including variables (retention time-mass-charge ratio), the observation volume (96 samples) and the integral area.

for a real-time correction of the $\mathrm{m} / \mathrm{z}$ values, the reference ion qualities were 121.050873 and 922.009798); mass spectrometry data recording mode, centroid mode; and instrument control and data acquisition software, MassHunter WorkStation Qualitative Analysis (Version B.03.01; Agilent Technologies, Inc.). Samples were analyzed at random using standard in multivarient data analysis (SIMCA)-P software version 11.0 (Umetrics, Umeå, Sweden). Group comparisons: B1 vs. A1; and B1 vs. D1.

Data processing. The total ion and base-peak chromatograms from the representative samples are shown in Fig. 1. MassHunter WorkStation Qualitative Analysis software (version B.03.01) was used to convert the raw data into the '*.mzdata' format. The $\mathrm{R}$ platform was then used to process the raw data with the XCMS package to obtain information about the baseline filter, peak identification, retention times and peak alignment. The three-dimensional structural data was then organized into a two-dimensional matrix of data, including variables (retention time-mass-charge ratio, rt_mz), the observation volume (96 samples) and the integral area. The XCMS centWave method was used to identify the peaks, peak width was set to 4-12 s, 30 parts per million (ppm); and the signal to noise ratio was set to 3 . The local regression (LOESS) method was used to perform a nonlinear correction to the retention time. The process observed 2,544 variables (rt_mz). Prior to performing statistical analysis, the total integrated area of each sample was normalized to 1,000 .

Statistical analysis. Significance was determined using the Student's paried t-test and the one-way analysis of variance on the mean of three different experiments. Statistical analysis was performed using SIMCA-P 11.0 software, which was standardized from the raw data to obtain an intuitive result. $\mathrm{P}<0.05$ was considered to indicate a statistically significant difference between values.

\section{Results}

Principal component analysis (PCA) compares B1 and Al. PCA was performed on the control and model groups using SIMCA-P software, which par-formatted and mean-centered the raw data for an intuitive result. The software automatically performed a model-fitting analysis for three principal components. The accumulated variance contribution rate was $\mathrm{R} 2 \mathrm{X}=0.572$. All samples were located within the $95 \%$ confidence interval, represented by the Hotelling's T2 ellipse (Fig. 2), which indicated that no outliers existed. In general, an $\mathrm{R} 2 \mathrm{X}$ value $>0.4$ indicates a reliable model, therefore the metabolic differences between the two sample groups determined by the present PCA model were reliable.

The results of the PCA revealed that samples from the control group were widely distributed throughout the PCA score plot, with the majority located in the upper quadrants; however, samples from the model group were limited to a small region of the score plot in the lower quadrants (Fig. 2). The two groups were not completely separated according to the PCA score plot; however, the decreased variability of the model group was significant enough to indicate that the model group was affected by induced COPD; other factors, including environmental, dietary and hereditary influences, had a rela- 
A1 vs B1, PCA-X, t[Comp. 1]tt[Comp. 2]

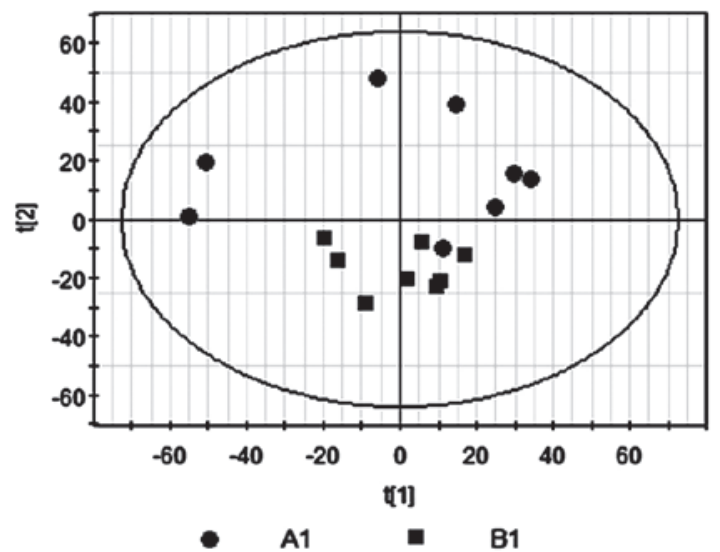

$R 2 X[1]=0.259593 \quad R 2 X[2]=0.201531 \quad$ Ellipse: Hotelling $T 2$ (0.95)

Figure 2. PCA score plot of A1 and B1. R2X=0.572. All samples were located within the $95 \%$ confidence interval, represented by the Hotelling's T2 ellipse, no outliers existed. PCA, principal component analysis; A1, healthy control group; B1, model group; R2X, accumulated variance contribution rate; $\mathrm{t}[1]$, first principle component score; $t[2]$, second principle component score.

A1 vs B1, PLS-DA, t[Comp. 1]t[Comp. 2]

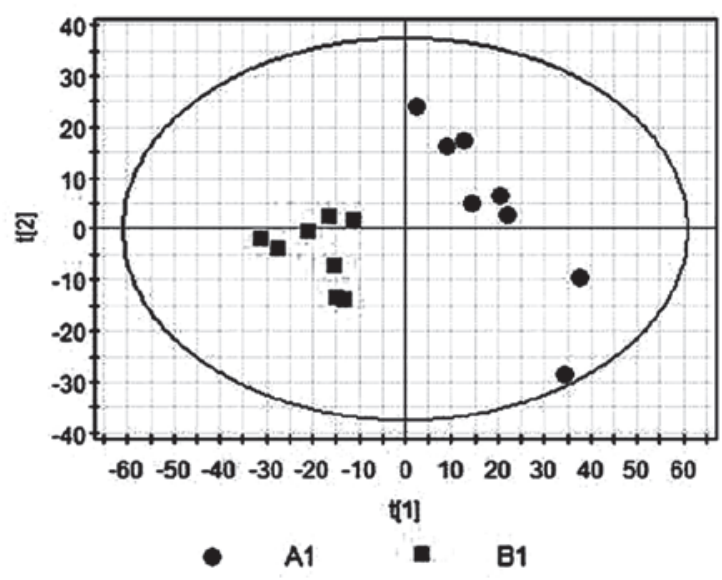

$R 2 \times[1]=0.193722 \quad R 2 \times[2]=0.0942006 \quad$ Ellipse: Hotelling $\mathrm{T} 2(0.95)$

Figure 3. PLS-DA score plot of A1 and B1. A total of three principal components were selected, as shown in the horizontal and verticle axes. PLS-DA, partial least squares-discriminant analysis; A1, healthy control group; $\mathrm{B} 1$, model group; $\mathrm{R} 2 \mathrm{X}$, accumulated variance contribution rate; $\mathrm{t}[1]$, first principle component score; $\mathrm{t}[2]$, second principle component score.

tively insignificant effect on the score plot. The separation trend of the PCA therefore indicated that there were significant metabolic differences between groups A1 and B1.

Partial least squares-discriminant analysis (PLS-DA) compares B1 and A1. PLS-DA, a supervisory multi-dimensional statistical model analysis method, was used to analyze the two sample groups. The software automatically filtered out noise. As shown in Fig. 3, a total of three principal components were selected for analysis, the first principal component score $t[1]$ is shown on the horizontal axis and the second principal component score $\mathrm{t}[2]$ is shown on the
A1 vs B1, OPLS-DA, t[Comp. 1]/t[Comp. 2]

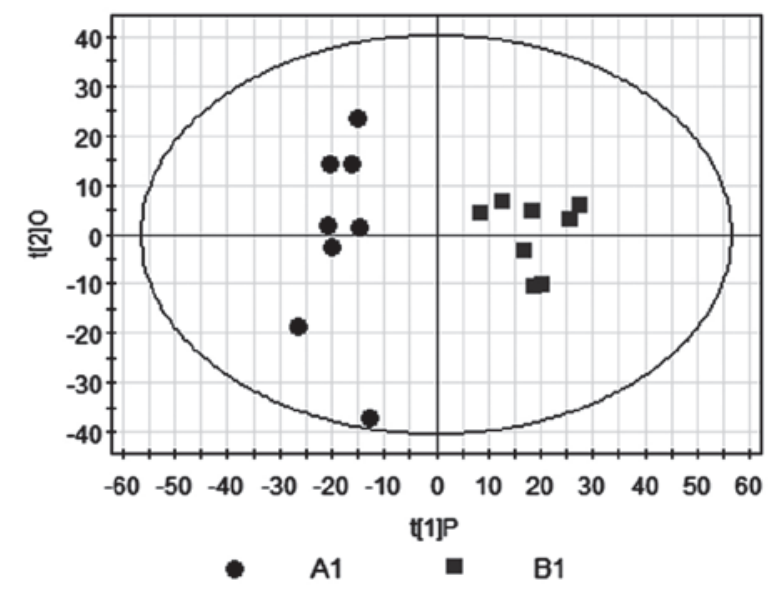

$R 2 X[1]=0.160646 \quad R 2 X[2]=0.127276 \quad$ Ellipse: Hotelling T2 (0.95)

Figure 4. OPLS-DA score plot of A1 and B1. Samples from B1 and A1 were separately distributed on the both sides of t[1]P. OPLS-DA, orthogonal partial least squares-discriminant analysis; A1, healthy control group; B1, model group; R2X, accumulated variance contribution rate; t[1]P, principle component score; $\mathrm{t}[2] \mathrm{O}$, quadrature component score.

vertical axis. The contribution rate of the supervision model, $\mathrm{R} 2 \mathrm{Y}=0.931$, indicated that PLS-DA was a reliable model for showing the differences between the two sample groups. The model forecast rate, $\mathrm{Q} 2=0.613$, demonstrated the predictive ability of the model. The results revealed that the control group was located in a larger area, while the model group was clustered in a smaller area. This therefore indicated that $\mathrm{A} 1$ and B1 had significant differences in their metabolic profile.

Orthogonal partial least squares-discriminant analysis (OPLS-DA) compares $B 1$ and $A 1$. In order to eliminate and classify noise in the control and model groups as well as to obtain more reliable significant differences in metabolite activity between the two groups, OPLS-DA was used to filter any irrelevant quadrature signals from the model classification. OPLS-DA consisted of one principal component (P), $\mathrm{R} 2 \mathrm{Y}=0.801$ and one quadrature component $(\mathrm{O}), \mathrm{R} 2 \mathrm{Y}=0.13$. The quality parameters of the model were $\mathrm{R} 2 \mathrm{Y}=0.931$ and $\mathrm{Q} 2=0.72$, indicating that the model was reliable. As shown in Fig. 4, the OPLS-DA score plot revealed comparable results to those of the PLS-DA model. The samples from the control and model groups were distributed on opposite sides of P; in addition, the control group was more sparsely spread across $\mathrm{O}$. This therefore indicated that the samples from the two groups had significant metabolic differences.

Identification of 49 metabolites with different concentrations in $B 1$ and $A 1$. Variable importance in projection (VIP; $>1$ ) was used to perform model analysis, in combination with t-test statistical analysis methods $(\mathrm{P}<0.05)$ to the explore different metabolites between the two groups. The molecular weights of the 49 metabolites that had different concentrations in B1 and A1 were identified according to the Metabolite and Tandem MS (Metlin) Database of the Human Metabolome 
Table I. The molecular weight of the 49 metabolites with different concentrations in B1 and A1 were identified according to the Metabolite and Tandem Mass Spectrometry Database of the Human Metabolome Database.

\begin{tabular}{|c|c|c|c|c|c|}
\hline Name & $\mathrm{rt}$ & $\mathrm{m} / \mathrm{z}$ & VIP(B1/A1) & metabolite & fold (B1/A1) \\
\hline M279T535_1 & 8.92 & 279.2320 & 2.10 & $\alpha$-Linolenic acid & 0.4862801 \\
\hline М369T354 & 5.90 & 369.2265 & 2.08 & 20-Hydroxy-PGE2 & 0.6286648 \\
\hline M281T535 & 8.91 & 281.2394 & 2.04 & Linoleic acid & 0.520539 \\
\hline M351T401 & 6.68 & 351.2163 & 1.90 & Lipoxin A5 & 0.475968 \\
\hline M213T466 & 7.76 & 213.1485 & 1.89 & 7-Oxo-11-dodecenoic acid & 0.5081364 \\
\hline M165T328 & 5.46 & 165.0905 & 1.72 & 3-Phenylbutyric acid & 0.3791378 \\
\hline M331T430 & 7.16 & 331.2667 & 1.71 & Eicosapentaenoic acid ethyl ester & 0.4811344 \\
\hline M214T302 & 5.03 & 214.1431 & 1.59 & $N$-Heptanoyl-homoserine lactone & 0.456305 \\
\hline M174T307 & 5.12 & 174.1117 & 1.56 & Acetyl-L-leucine & 0.502141 \\
\hline M337T492 & 8.21 & 337.2370 & 1.51 & 11-Deoxy-PGE2 & 0.4664266 \\
\hline M165T305 & 5.08 & 165.0595 & 1.37 & Phenylpyruvic acid & -0.683227 \\
\hline M165T318 & 5.29 & 165.0755 & 1.21 & $\alpha$-D-Fucose & 0.3453485 \\
\hline M398T484 & 8.07 & 398.2902 & 1.21 & PGE1 ethanolamide & 0.5641619 \\
\hline M319T535 & 8.91 & 319.2247 & 2.08 & 5-hydroxyeicosapentaenoic acid & 0.5407731 \\
\hline М367Т397 & 6.62 & 367.2451 & 1.84 & PGE2 methyl ester & 0.4350498 \\
\hline М303Т316 & 5.27 & 303.1911 & 1.81 & 2-Methoxyestradiol & 0.5379153 \\
\hline M321T585 & 9.76 & 321.2420 & 1.81 & 5-Hydroxyeicosatetraenoic acid & 0.4598037 \\
\hline M335T455 & 7.58 & 335.2199 & 1.78 & 20-Carboxy arachidonic acid & 0.4121728 \\
\hline M335T369 & 6.14 & 335.2215 & 1.78 & 20-Carboxy arachidonic acid & 0.4440207 \\
\hline M311T543 & 9.06 & 311.2220 & 1.71 & $13(S)-\mathrm{HpOTrE}$ & 0.6599862 \\
\hline M333T543 & 9.05 & 333.2040 & 1.69 & $11 \beta$-hydroxyprogesterone & 0.5746297 \\
\hline M338T586 & 9.77 & 338.2695 & 1.67 & Linoleoyl glycine & \\
\hline M285T800 & 13.33 & 285.2798 & 1.67 & Stearic acid & -0.290979 \\
\hline M208T310 & 5.16 & 208.0964 & 1.65 & $N$-Acetyl-L-phenylalanine & 0.4514552 \\
\hline M353T363 & 6.05 & 353.2300 & 1.65 & 20-Hydroxy LTB4 & 0.3555726 \\
\hline M126T298 & 4.97 & 126.0219 & 1.64 & Taurine & 0.5644918 \\
\hline M367T352 & 5.86 & 367.2114 & 1.63 & 20-Carboxy-LTB4 & 0.4634855 \\
\hline M205T328 & 5.47 & 205.0832 & 1.63 & $\gamma$-D-Glutamylglycine & 0.4171233 \\
\hline M242T310 & 5.177 & 242.1745 & 1.61 & $\mathrm{~N}$-Nonanoyl-L-Homoserine lactone & 0.4906304 \\
\hline М289T307 & 5.12 & 289.1752 & 1.60 & 2-Hydroxyestradiol & 0.5220455 \\
\hline М307T606 & 10.11 & 307.2627 & 1.58 & Linolenic acid ethyl ester & 0.6468999 \\
\hline M305T578 & 9.63 & 305.2476 & 1.51 & Arachidonic acid (peroxide free) & 0.3752692 \\
\hline M313T363 & 6.04 & 313.2373 & 1.49 & $13(S)-\mathrm{HpODE}$ & 0.364127 \\
\hline M271T315 & 5.25 & 271.1645 & 1.49 & Estrone & 0.4819101 \\
\hline M213T362 & 6.04 & 213.1483 & 1.48 & 7-Oxo-11-dodecenoic acid & 0.3521852 \\
\hline M136T49 & 0.82 & 136.0756 & 1.48 & Phenacylamine & 0.1746112 \\
\hline М327Т699 & 11.66 & 327.2296 & 1.46 & 4,5-Dehydro docosahexaenoic acid & -0.611621 \\
\hline M295T363 & 6.04 & 295.2268 & 1.43 & 13(S)-HOTrE & 0.3480763 \\
\hline M149T316 & 5.27 & 149.0448 & 1.40 & D- $\alpha$-Hydroxyglutaric acid & 0.2677423 \\
\hline M297T566 & 9.43 & 297.2350 & 1.38 & 9-hydroxy-10,12-octadecadienoic acid & 0.5084189 \\
\hline M518T527 & 8.78 & 518.3248 & 1.37 & LysoPC(18:3) & -0.695892 \\
\hline M160T303 & 5.05 & 160.1327 & 1.33 & 2-Amino butanoic acid & 0.3999979 \\
\hline M333T389 & 6.49 & 333.2417 & 1.28 & Leukotriene A4 methyl ester & 0.1849361 \\
\hline M528T505 & 8.41 & 528.2997 & 1.25 & LysoPE(22:5) & 0.5568003 \\
\hline M175T44 & 0.74 & 175.0232 & 1.24 & Aconitic acid & 0.7021798 \\
\hline М300T269 & 4.49 & 300.0949 & 1.21 & 8-Hydroxy guanosine & -0.462865 \\
\hline M277T564 & 9.40 & 277.2164 & 1.20 & 9,12-Octadecadienoic acid & 0.4366534 \\
\hline М322Т699 & 11.66 & 322.2742 & 1.14 & $\alpha$-Linolenoyl ethanolamide & -0.570115 \\
\hline М232Т288 & 4.80 & 232.1542 & 1.11 & Isobutyryl carnitine & 0.463761 \\
\hline
\end{tabular}

Threshold of quality, 30 parts per million; rt, retention time; m/z, mass charge ratio; VIP, variable importance in projection; A1, healthy control group; B1, model group; LTB4, leukotriene B4; PGE, prostaglandin E; HpODE, hydroperoxyoctadeca-9Z,11E-dienoic acid; $\mathrm{H}(\mathrm{p}) \mathrm{OTrE}$, hydro(pero)xy-9Z,11E,15Z-octadecatrienoic acid; LysoPC/PE, lysophosphatidyl choline/ethanolamine. 
D1 vs B1, PCA-X, t[Comp. 1]/t[Comp. 2]

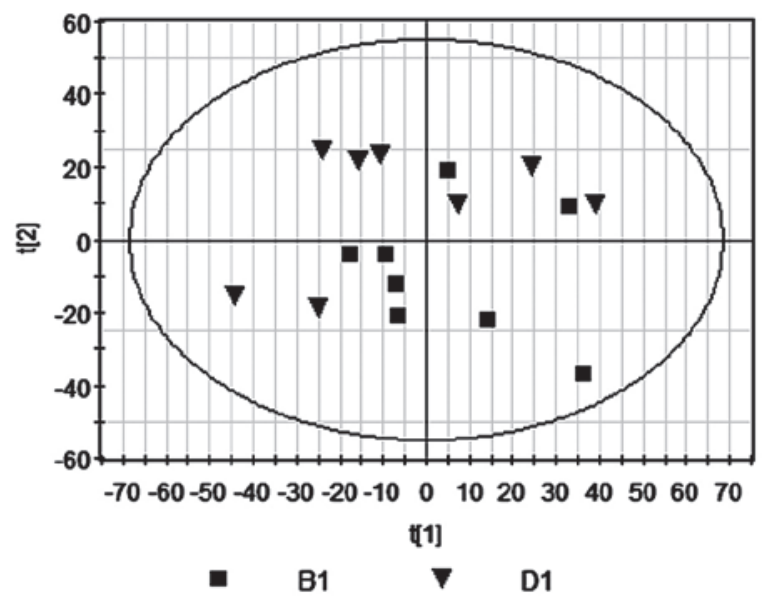

$R 2 X[1]=0.236142 \quad R 2 X[2]=0.150892 \quad$ Ellipse: Hotelling T2 (0.95)

Figure 5. PCA score plot of B1 and D1. R2X=0.508. All samples were located within the $95 \%$ confidence interval, represented by the Hotelling's T2 ellipse, no outliers existed. PCA, principal component analysis; B1, model group; $\mathrm{D} 1$, Bu-fei Jian-Pi-treated group; R2X, accumulated variance contribution rate; $\mathrm{t}[1]$, first principle component score; $\mathrm{t}[2]$, second principle component score.

\section{D1 vs B1, PLS-DA, t[Comp. 1]/t[Comp. 2]}

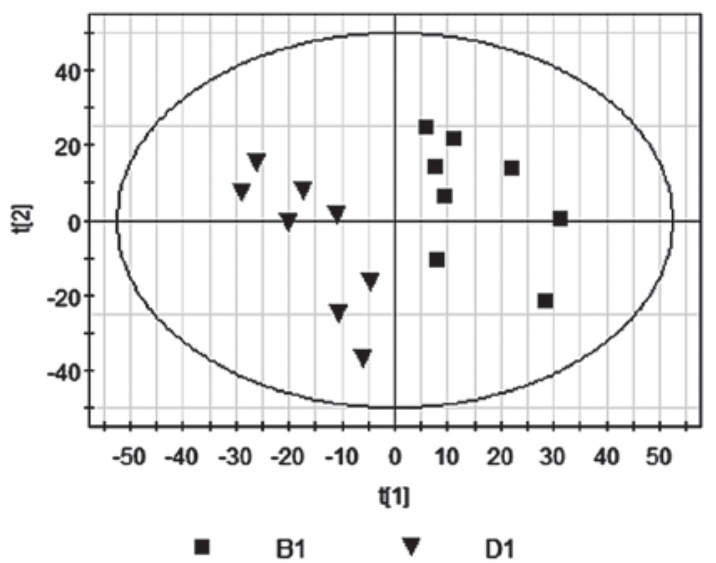

$R 2 X[1]=0.158763 \quad R 2 X[2]=0.176157 \quad$ Ellipse: Hotelling $T 2$ (0.95)

Figure 6. PLS-DA score plot of B1 and D1. PLS-DA score plot of B1 and D1. A total of three principal components were selected, as shown in the horizontal and verticle axes. PLS-DA, partial least squares-discriminant analysis; B1, model group; D1, Bu-Fei Jian-Pi-treated group; R2X, accumulated variance contribution rate; t[1], first principle component score; $t[2]$, second principle component score.

Database (HMDB; http://metlin.scripps.edu/index.php). The threshold for quality was $30 \mathrm{ppm}$ (Table I).

PCA compares BI and D1. PCA was performed on rats in the Bu-Pi Jian-Fei-treated and model groups using SIMCA-P software under identical conditions to those of the PCA of B1 and A1. The accumulated variance contribution rate was $\mathrm{R} 2 \mathrm{X}=0.508$, therefore indicating that the PCA model was reliable. As shown in Fig. 5, all samples in the PCA score plot were located within the $95 \%$ confidence interval, represented
D1 vs B1, OPLS-DA, t[Comp. 1]/t[Comp. 2]

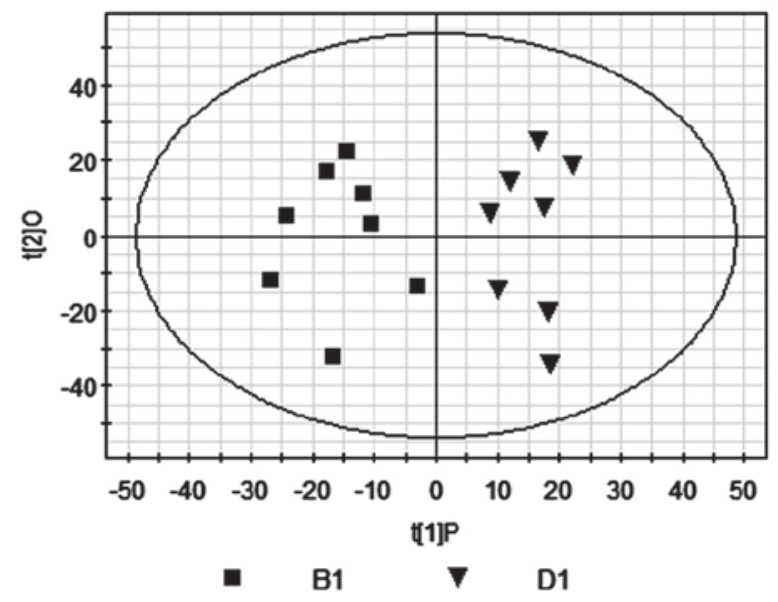

$R 2 X[1]=0.126019 \quad R 2 X[2]=0.208901 \quad$ Ellipse: Hotelling T2 (0.95)

Figure 7. OPLS-DA score plot of B1 and D1. Samples from B1 and D1 were separately distributed on both sides of t[1]P. OPLS-DA, orthogonal partial least squares-discriminant analysis; B1, model group; D1, Bu-Fei Jian-Pi-treated group; R2X, accumulated variance contribution rate; $\mathrm{t}[1] \mathrm{P}$, principle component score; $\mathrm{t}[2] \mathrm{O}$, quadrature component score.

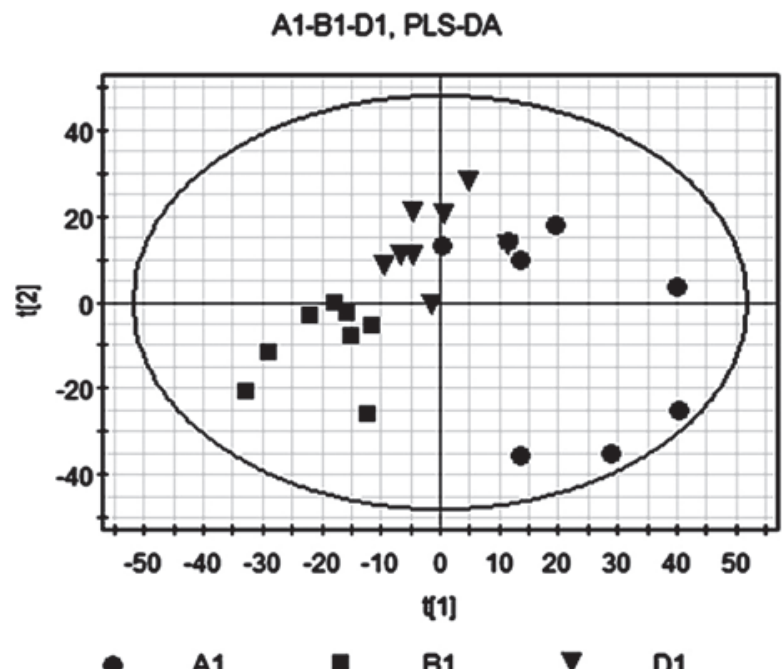

Figure 8. PLS-DA score plot of A1, B1 and D1. A total of three principal components were selected, as shown in the horizontal and verticle axes. PLS-DA, partial least squares-discriminant analysis; B1, model group; D1, Bu-Fei Jian-Pi-treated group; t[1], first principle component score; t[2], second principle component score.

by the Hotelling's T2 ellipse, which indicated that no outliers were present. The results showed no significant separation between the two groups; however, there was a relatively weak separation trend as the distribution of B1 tended to locate in the lower right quadrant and D1 in the upper left quadrant.

PLS-DA compares B1 and D1. PLS-DA was used as described in the PLS-DA comparison of $\mathrm{B} 1$ and A1. The contribution rate of supervision model, R2Y=0.998, indicated that PLS-DA was a reliable model for the differences between the two sample groups and the model forecast rate, Q2 $=0.859$ demonstrated the predictive ability of the model. As shown in Fig. 6, the sepera- 
Table II. The molecular weight of the 31 different metabolites between B1 and D1 were identified according to the Metabolite and Tandem Mass Spectometry Database of the Human Metabolome Database.

\begin{tabular}{|c|c|c|c|c|c|}
\hline Name & $\mathrm{rt}$ & $\mathrm{m} / \mathrm{z}$ & VIP(B1/D1) & metabolite & fold (D1/B1) \\
\hline M165T305 & 5.08 & 165.0595 & 2.27 & Phenylpyruvic acid & 0.24 \\
\hline M476T445 & 7.41 & 476.2646 & 2.26 & LysoPE (18:3) & 0.21 \\
\hline M369T354 & 5.90 & 369.2265 & 2.20 & 20-Hydroxy-PGE2 & -0.36 \\
\hline M536T415 & 6.92 & 536.3341 & 2.14 & LysoPC (18:1) & -0.40 \\
\hline M337T492 & 8.21 & 337.2370 & 2.08 & Leukotriene B4 & -0.46 \\
\hline M273T318 & 5.30 & 273.1801 & 2.06 & $\beta$-Estradiol & -0.55 \\
\hline M279T535_1 & 8.92 & 279.2320 & 2.05 & $\gamma$-Linolenic acid & -0.21 \\
\hline M496T548 & 9.14 & 496.3326 & 2.05 & LysoPC(16:0) & -0.39 \\
\hline M281T535 & 8.91 & 281.2394 & 2.03 & Linoleic acid & -0.38 \\
\hline M147T317 & 5.29 & 147.0649 & 1.98 & Adipic acid & 0.30 \\
\hline M330T460 & 7.67 & 330.2641 & 1.98 & 4,8 Dimethylnonanoyl carnitine & -0.26 \\
\hline M147T48 & 0.81 & 147.0440 & 1.97 & Phenylpropiolic acid & -0.34 \\
\hline M137T51_1 & 0.85 & 137.0459 & 1.96 & Hypoxanthine & 0.40 \\
\hline M351T485 & 8.09 & 351.2164 & 1.94 & Lipoxin A5 & -0.33 \\
\hline М508Т608 & 10.13 & 508.3330 & 1.91 & LysoPE (20:1) & 0.24 \\
\hline M147T804 & 13.40 & 147.0653 & 1.90 & Adipic acid & 0.61 \\
\hline M355T526 & 8.76 & 355.2842 & 1.87 & 1-Linoleoyl glycerol & -0.82 \\
\hline M398T484 & 8.07 & 398.2902 & 1.85 & PGE1 ethanolamide & -0.84 \\
\hline M214T302 & 5.03 & 214.1431 & 1.83 & $N$-Heptanoyl-homoserine lactone & -0.37 \\
\hline M331T430 & 7.16 & 331.2667 & 1.82 & Eicosapentaenoic acid ethyl ester & -0.31 \\
\hline M295T429 & 7.15 & 295.2268 & 1.82 & 9-Oxooctadeca-10,12-dienoic acid & -0.24 \\
\hline M174T307 & 5.12 & 174.1117 & 1.78 & Acetyl-L-leucine & -0.39 \\
\hline M213T466 & 7.76 & 213.1485 & 1.78 & 7-Oxo-11-dodecenoic acid & -0.22 \\
\hline M551T606 & 10.14 & 551.3555 & 1.78 & Phosphatidic acid (25:0) & 0.23 \\
\hline M165T318 & 5.29 & 165.0755 & 1.72 & $\alpha-\mathrm{D}-\mathrm{Fucose}$ & 0.24 \\
\hline M351T401 & 6.68 & 351.2163 & 1.70 & Lipoxin A5 & -0.19 \\
\hline M293T417 & 6.96 & 293.2109 & 1.69 & 9-Oxo-10,12,15-octadecatrienoic acid & -0.38 \\
\hline M126T38 & 0.64 & 126.0637 & 1.69 & 5-Methylcytosine & 0.62 \\
\hline M428T40 & 0.67 & 428.0368 & 1.65 & Adenosine diphosphate & 0.20 \\
\hline M165T328 & 5.46 & 165.0905 & 1.60 & 3-Phenylbutyric acid & -0.18 \\
\hline M383Т569 & 9.48 & 383.2796 & 1.32 & 20-Ethyl prostaglandin $\mathrm{F} 2 \alpha$ & 0.21 \\
\hline
\end{tabular}

Threshold of quality, 30 parts per million; rt, retention time; m/z, mass charge ratio. VIP, variable importance in projection; B1, model group; D1, Bu-Fei Jian-Pi-treated group. PGE, prostaglandin E; LysoPC/PE, lysophosphatidyl choline/ethanolamine.

tion of the model and Bu-Fei Jian-Pi-treated groups on opposite sides of the horizontal axis indicated that the metabolic activity of rats in the D1 group was significantly altered following treatment.

OPLS-DA compares B1 and D1. OPLS-DA was performed as described in the OPLS-DA comparison of B1 and A1. The $\mathrm{P}$ and $\mathrm{O}$ were $\mathrm{R} 2 \mathrm{Y}=0.75$ and $\mathrm{R} 2 \mathrm{Y}=0.123$, respectively. The quality parameters of the model were $\mathrm{R} 2 \mathrm{Y}=0.874$ and $\mathrm{Q} 2=0.565$. Scores are shown in Fig. 7. Similarly to the results of the PLS-DA model, the OPLS-DA model explained that R2Y and the predicted rate Q2 were high, indicating that the current model was reliable. As shown in Fig. 7, the OPLS-DA score plot revealed comparable results to those of the PLS-DA model. The samples from the model and $\mathrm{Bu}-\mathrm{Fei}$ Jian-Pi-treated groups were distributed on opposite sides of $\mathrm{P}$. This therefore indicated that there were significant metabolic differences between the two groups.

Identification of the different metabolites between B1 and D1. $\operatorname{VIP}(>1)$ and t-tests were used to perform model and statistical analysis $(\mathrm{P}<0.05)$ of the different metabolites between the two groups. The molecular weights of the 31 different metabolites was identified according to the Metlin Database, with a threshold of quality of $30 \mathrm{ppm}$ (Table II).

PLS-DA of A1, B1 and D1. PLS-DA was used as previously described in the PLS-DA comparison of B1 and A1. The contribution rate of supervision model was $\mathrm{R} 2 \mathrm{Y}=0.998$, which indicated that PLS-DA was a reliable model for the differences between the two sample groups and the model forecast rate $\mathrm{Q} 2=0.859$ demonstrated the predictive ability of the model. 
Table III. Identification of 13 metabolites with different concentration among A1, B1 and D1 according to the Metabolite and Tandem Mass Spectometry Database of the Human Metabolome Database.

\begin{tabular}{|c|c|c|c|c|c|c|c|}
\hline Name & $\mathrm{rt}$ & $\mathrm{m} / \mathrm{z}$ & metabolite & $\begin{array}{c}\text { VIP } \\
(\mathrm{B} 1 / \mathrm{A} 1)\end{array}$ & $\begin{array}{c}\text { fold } \\
\text { (B1/A1) }\end{array}$ & $\begin{array}{c}\text { VIP } \\
\text { (B1/D1) }\end{array}$ & $\begin{array}{c}\text { fold } \\
\text { (D1/B1) }\end{array}$ \\
\hline M279T535_1 & 8.92 & 279.23 & $\alpha$-Linolenic acid & 2.10 & 0.49 & 2.05 & -0.21 \\
\hline М369T354 & 5.90 & 369.23 & 20-Hydroxy-PGE2 & 2.08 & 0.63 & 2.20 & -0.36 \\
\hline M281T535 & 8.91 & 281.24 & Linoleic acid & 2.04 & 0.52 & 2.03 & -0.38 \\
\hline M351T401 & 6.68 & 351.22 & Lipoxin A5 & 1.90 & 0.48 & 1.70 & -0.19 \\
\hline M213T466 & 7.76 & 213.15 & 7-Oxo-11-dodecenoic acid & 1.89 & 0.51 & 1.78 & -0.22 \\
\hline M165T328 & 5.46 & 165.09 & 3-Phenylbutyric acid & 1.72 & 0.38 & 1.60 & -0.18 \\
\hline M331T430 & 7.16 & 331.27 & Eicosapentaenoic acid ethyl ester & 1.71 & 0.48 & 1.82 & -0.31 \\
\hline M214T302 & 5.03 & 214.14 & $\mathrm{~N}$-Heptanoyl-homoserine lactone & 1.59 & 0.46 & 1.83 & -0.37 \\
\hline M174T307 & 5.12 & 174.11 & Acetyl-L-leucine & 1.56 & 0.50 & 1.78 & -0.39 \\
\hline M337T492 & 8.21 & 337.24 & 11-Deoxy-PGE2 & 1.51 & 0.47 & 2.08 & -0.46 \\
\hline M165T305 & 5.08 & 165.06 & Phenylpyruvic acid & 1.37 & 0.68 & 2.27 & 0.24 \\
\hline M165T318 & 5.29 & 165.08 & $\alpha$-D-Fucose & 1.21 & 0.35 & 1.72 & 0.24 \\
\hline M398T484 & 8.07 & 398.29 & PGE1 ethanolamide & 1.21 & 0.56 & 1.85 & -0.84 \\
\hline
\end{tabular}

Threshold of quality, 30 parts per million; rt, retention time; m/z, mass charge ratio; VIP, variable importance in projection; A1, healthy control group; B1, model group; D1, Bu-fei Jian-Pi-treated group. PGE, prostaglandin E.

As shown in Fig. 8, the model group in the right quadrants and the Bu-Fei Jian-Pi-treated group in the left quadrants indicated that rats in the D1 group had a significantly different metabolic profile from that of rats in the untreated group.

Identification of metabolites with different concentrations in A1, B1 and D1. Following treatment with Bu-Fei Jian-Pi, 13 metabolites had different concentrations among the three groups. Eleven metabolites showed a negative fold change in the Bu-Fei Jian-Pi-treated group compared to that of the model group; however, minimal changes were observed in phenylpyruvic acid and $\alpha$-D-Fucose expression (Table III).

\section{Discussion}

COPD is a prevalent disease with high rates of mortality (1). COPD is defined by the persistent and increasing limitation of airflow, which may be enhanced by exacerbations and the occurence of additional morbidity in patients (2). Current treatment of COPD uses conventional Western classes of medication proposed by the Global Initiative for Chronic Obstructive Lung Diseases (GOLD) (3). However, TCM has been used for numerous years as an alternative method of treatment for COPD patients. The present study aimed to elucidate the molecular mechanisms of COPD via metabolomic analysis and to explore the targets and intervention mechanisms of the common TCM granules Bu-Fei Jian-Pi in a rat model of stable COPD.

In the present study Bu-Fei Jian-Pi granules were produced from a selection of herbs according to a traditional Chinese formula (5). Treatment was performed over three months followed by a further three months observation of the rats. The results revealed favourable effects on COPD model rats, with no adverse events.
Metabonomics is a technique used in pharmaceutical and clincal studies for rapidly analyzing biological dysfunction. It may be used in conjunction with nuclear magnetic resonance (NMR) of biological fluids using high resolution ${ }^{1} \mathrm{H}$ spectroscopic profiling and multivariate analyis in order to identify metabolic changes associated with physiological conditions $(10,11)$.

Metabonics is defined as the quantitative measurement of the response of living systems in dynamic multi-parametric conditions to genetic modification or pathophysiological stimuli (12). It provides an overal quantification of the low-molecular endogenous metabolites found in biological samples, including tissue, urine and plasma $(13,14)$. Data sets provided by high-resolution ${ }^{1} \mathrm{H}$ NMR spectroscopy are complex and require chemometrics approaches in order to visulize them and identify patterns in the spectral measurements. Metabolites may therefore be used to classify models for numerous disease states, toxic shock, genetic modifications and dietary changes (15-19). PCA, SIMCA, PLS-DA and neural networks are amongst the most frequently used chemometric approaches for analyzing metabonomics data $(13,18,20-22)$.

In the present study, PCA models were used in order to explain the metabolic differences between the control and model groups. The results demonstrated a significantly reduced variablilty of the model group compared to that of the control group; in addition, the separation trend along the vertical axis was observably different between the two groups. This therefore indicated that there was a significant difference between the metabolic profiles of the model (B1) and control groups (A1). Furthermore, the PLA-DA model showed that following noise filtration, A1 and B1 had significant metabolic differences and the OPLS-DA model demonstrated that samples from the model and control 
groups were separately distributed on the opposite sides of the first principal component (PC1). This provided further evidence for the significant metabolic differences between the control and model groups. The molecular weights of the 49 differentially concentrated metabolites were then identified according to the Metlin Database.

PCA models demonstrated that there was a weak, but not significant separation trend between the model and Bu-Fei Jian-Pi-treated groups. However, PLS-DA models indicated that following treatment with Bu-Fei Jian-Pi, COPD rats had significantly different metabolic profiles from those in the model group. In addition, the results of the OPLS-DA revealed that the samples from the model and the $\mathrm{Bu}-\mathrm{Fei}$ Jian-Pi-treated groups were distributed on opposite sides of the PC1, indicating that the two groups had significant metabolic differences. The molecular weights of the 31 differentially concentrated metabolites between the model and $\mathrm{Bu}-\mathrm{Fe}$ Jian-Pi groups were then identified according to the Metlin Database.

Following treatment with Bu-Fei Jian-Pi, 13 metabolites had the same B1/A1 and B1/D1 ratio, indicating that metabolite levels in mice of the B1 group were restored to those of healthy mice (A1 group) following treatment. Eleven metabolites showed a negative fold change in the Bu-Fei Jian-Pi-treated groups compared to levels in the model group; however, minimal changes were observed in phenylpyruvic acid and $\alpha$-D-fucose levels.

COPD has an unpredictable clinical course with a diverse range of phenotypes; as a result, its progression and development remain difficult to determine and there have been no clearly defined biomarkers (7). However, previous studies have shown that the use of NMR and MS, together with chemometric analysis, may be used to evaluate the metabolic activities of biological systems. This may therefore be used as a diagnostic tool for metabolomic analysis, with the ability to observe qualitative and quantitative changes in cellular metabolism (11). Furthermore, these techniques may lead to the detection of homeostatic disturbances prior to clincical manifestations of diseases (12). There have been few previous studies into the metabolic analysis of COPD; however, it has been suggested that these metabolomic methods have the potential to distinguish COPD from other diseases as well as diagnose COPD, including its classification and stage of progression (23).

In conclusion, the results of the present study demonstrated significant metabolic differences in the lungs of COPD model rats [constructed by smoke inhalation and recurrent bacterial infection $(24,25)]$ compared with those of healthy control rats. In addition, COPD model rats treated with the TCM Bu-Fei Jian-Pi granules were revealed to have a significantly different spectrum of metabolites from that of untreated COPD rats. This therefore indicated that TCM had a significant beneficial effect in a rat model of stable COPD. Furthermore, the significantly altered metabolite profile of COPD rat models compared to that of the control rats may provide evidence for potential COPD biomarkers. However, further studies are required in order to assess the sensitivity and specificity of these methods in COPD as well as to evaluate TCM treatment on COPD via these specific potential biomarkers.

\section{Acknowledgements}

This study was supported by grants from the National Natural Science Foundation of China (no. 81130062).

\section{References}

1. Raherison C and Girodet PO: Epidemiology of COPD. Eur Respir Rev 18: 213-221, 2009.

2. Lin HH, et al: Effects of smoking and solid-fuel use on COPD, lung cancer, and tuberculosis in China: a time-based, multiple risk factor, modelling study. Lancet 372: 1473-1483, 2008.

3. Qaseem A, Wilt TJ, Weinberger SE, et al; American college of Physicians; American Collage of Chest Physicians; American Thoracic Society and European Respiratory Society: Diagnosis and management of stable chronic obstructive pulmonary disease: a clinical practice guideline update from the American College of Physicians, American College of Chest Physicians, American Thoracic Society, and European Respiratory Society. Ann Intern Med 155: 179-191, 2011.

4. Calverley PM, Anderson JA, Celli B, et al: Satmeterol and flutieasene propionate and survival in chronic obstructive pulmonary disease. N Engl J Med 356: 775-789, 2007.

5. Li JS, Wang ZW, Yu XQ, et al: Clinical efficacy and safety of TCM for COPD at stable phase: A Systematic review. Traditional Chinese Medicine of Liaoning 37: 229-232, 2010.

6. Li JS, Wang ZW, Yu XQ, et al: Systemic evaluation of TCM for COPD in the acute stage of exacerbation. Traditional Chinese Medicine of Tianjin 25: 428-431, 2008.

7. Zhang WJ and Zhang YP: Recent research in chronic obstructive pulmonary disease treated with TCM. Clinical Traditional Chinese Medicine of Beijing 14: 39-41, 2007.

8. Lu AP and Chen KJ: Chinese medicine pattern diagnosis could lead to innovation in medical sciences. Chin J Integr Med 17: 811-817, 2011.

9. Wang ZW, Li JS, et al: Symptom diagnosis criteria for chronic obstructive pulmonary diseases at stationary phase from literature. The Science of Traditional Chinese Medicine 218: 55-58, 2008.

10. Nicholls AW, Nicholson JK, Haselden JN and Waterfield CJ: A metabonomic approach to the investigation of drug-induced phospholipidosis: an NMR spectroscopy and pattern recognition study. Biomarkers 5: 410-423, 2000.

11. Nicholson JK and Wilson ID: Understanding 'global' systems biology: metabonomics and the continuum of metabolism. Nat Rev Drug Discov 2: 668-676, 2003.

12. Nicholson JK, Lindon JC and Holmes E: 'Metabonomics': understanding the metabolic responses of living systems to pathophysiological stimuli via multivariate statistical analysis of biological NMR spectroscopic data. Xenobiotica 29: 1181-1189, 1999.

13. Holmes E, Nicholls AW, Lindon JC, et al: Chemometric models for toxicity classification based on NMR spectra of biofluids. Chem Res Toxicol 13: 471-478, 2000.

14. Lindon JC, Nicholson JK, Holmes E, et al: Metabonomics: Metabolic processes studied by NMR spectroscopy of biofluids. Concepts Magn Reson 12: 289-320, 2000.

15. Brindle JT, Antti H, Holmes E, et al: Rapid and noninvasive diagnosis of the presence and severity of coronary heart disease using 1H-NMR-based metabonomics. Nat Med 8: 1439-1444, 2002.

16. Keun HC, Ebbels TM, Bollard ME, et al: Geometric trajectory analysis of metabolic responses to toxicity can define treatment specific profiles. Chem Res Toxicol 17: 579-587, 2004.

17. Lindon JC, Nicholson JK, Holmes E, et al: Contemporary issues in toxicology the role of metabonomics in toxicology and its evaluation by the COMET project. Toxicol Appl Pharmacol 187: 137-146, 2003.

18. Holmes E, Nicholson JK and Tranter G: Metabonomic characterization of genetic variations in toxicological and metabolic responses using probabilistic neural networks. Chem Res Toxicol 14: 182-191, 2001.

19. Solanky KS, Bailey NJ, et al: Application of biofluid $1 \mathrm{H}$ nuclear magnetic resonance-based metabonomic techniques for the analysis of the biochemical effects of dietary isoflavones on human plasma profile. Anal Biochem 323: 197-204, 2003. 
20. Holmes $\mathrm{E}$ and Antti $\mathrm{H}$ : Chemometric contributions to the evolution of metabonomics: mathematical solutions to characterising and interpreting complex biological NMR spectra. Analyst 127: 1549-1557, 2002.

21. Gavaghan CL, Wilson ID and Nicholson JK: Physiological variation in metabolic phenotyping and functional genomic studies: use of orthogonal signal correction and PLS-DA. FEBS Lett 530: 191-196, 2002.

22. Ebbels T, Keun H, Beckonert O, et al: Toxicity classification from metabonomic data using a density superposition approach: 'CLOUDS' Anal Chim Acta 490: 109-122, 2003.
23. Młynarz P, Barg W, Deja S and Jankowska R: Application of metabolomic in COPD diagnosing. Pol Merkur Lekarski 33: 207-212, 2012 (In Polish)

24. Li Y, Li SY, Li JS, et al: A rat model for stable chronic obstructive pulmonary disease induced by cigarette smoke inhalation and repetitive bacterial infection. Biol Pharm Bull 35: 1752-1760, 2012. 